\title{
THE CLASSICAL TRILOGARITHM, ALGEBRAIC $K$-THEORY OF FIELDS, AND DEDEKIND ZETA FUNCTIONS
}

\author{
A. B. GONCHAROV
}

\begin{abstract}
In this paper we show how to express the values of $\zeta_{F}(3)$ for arbitrary number field $F$ in terms of the trilogarithms (D. Zagier's conjecture) and how to relate this result to algebraic $K$-theory.
\end{abstract}

\section{THE ClASSICAL POLYLOGARITHM FUNCTION}

The classical polylogarithm function

$$
\operatorname{Li}_{p}(z):=\sum_{n=1}^{\infty} \frac{z^{n}}{n^{p}}(z \in \mathbf{C},|z| \leq 1, p \in \mathbf{N})
$$

during the last 200 years was the subject of much research-see [L]. Using the inductive formula $\mathrm{Li}_{p}(z)=\int_{0}^{z} \mathrm{Li}_{p-1}(t) t^{-1} d t, \mathrm{Li}_{1}(z)=$ $-\log (1-z)$, the $p$-logarithm can be analytically continued to a multivalued function on $\mathbf{C} \backslash\{0,1\}$. However, D. Wigner and $\mathbf{S}$. Bloch introduced [B1] the single-valued cousin of the dilogarithm, namely

$$
D_{2}(z):=\operatorname{Im}\left(\operatorname{Li}_{2}(z)\right)+\arg (1-z) \cdot \log |z| .
$$

Of course, for $\mathrm{Li}_{1}$ such function is $-\log |z|$. Analogous functions $D_{p}(z)$ for $p \geq 3$ were introduced in $[\mathrm{R}]$ and computed explicitly in [Z]. Let us consider the slightly modified function

$$
\mathscr{L}_{3}(z):=\operatorname{Re}\left[\operatorname{Li}_{3}(z)-\log |z| \cdot \operatorname{Li}_{2}(z)+\frac{1}{3} \log ^{2}|z| \cdot \operatorname{Li}_{1}(z)\right] .
$$

Such modified functions were considered also for all $p$ by D. Zagier, A. A. Beilinson and P. Deligne [Z3, Be1]. $\mathscr{L}_{3}(z)$ is real-analytic on $\mathbf{C} P^{1} \backslash\{0,1, \infty\}$ and continuous on $\mathbf{C} P^{1}$.

Let $F$ be a field. Let $P_{F}^{1}$ be the projective line over $F$, and let $\mathbf{Z}\left[P_{F}^{1} \backslash 0,1, \infty\right]$ be the free abelian group generated by symbols $\{x\}$, where $x \in P_{F}^{1}\{0,1, \infty\}$.

Received by the editors February 9, 1990 and, in revised form, June 15, 1990. 1980 Mathematics Subject Classification (1985 Revision). Primary 19F27,11F67. The proofs for this paper were reviewed by the editor. 
We may consider $\mathscr{L}_{3}$ as defining a homomorphism

$$
\mathscr{L}_{3}: \mathbf{Z}\left[P_{\mathbf{C}}^{1} \backslash 0,1, \infty\right] \rightarrow \mathbf{R}, \quad \mathscr{L}_{3}: \Sigma n_{i}\left\{x_{i}\right\} \mapsto \Sigma n_{i} \mathscr{L}_{3}\left(x_{i}\right) .
$$

We can do the same for any other real-valued function on $P_{\mathbf{C}}^{1} \backslash\{0,1, \infty\}$, in particular for $D_{2}$.

\section{FORMULA FOR $\zeta(3)$}

Now let $F$ be an arbitrary algebraic number field, $d_{F}$ the discriminant of $F, r_{1}$ and $r_{2}$ the number of real and complex places, $\sigma_{j}$ all possible embeddings $F \hookrightarrow \mathbf{C}, 1 \leq j \leq r_{1}+2 r_{2}$, and $\overline{\sigma_{r_{1}+k}}=\sigma_{r_{1}+r_{2}+k}$. Set $A_{\mathbf{Q}}:=A \otimes \mathbf{Q}$. Let us consider the homomorphism

$$
\begin{gathered}
\Delta: \mathbf{Q}\left[P_{F}^{1} \backslash 0,1, \infty\right] \rightarrow\left(\Lambda^{2} F^{*} \otimes F^{*}\right)_{\mathbf{Q}}, \\
\Delta:\{x\} \mapsto(1-x) \wedge x \otimes x .
\end{gathered}
$$

Theorem 1. Let $\zeta_{F}(s)$ be the Dedekind zeta function of $F$. Then there exist $y_{1}, \ldots, y_{r_{1}+r_{2}} \in \operatorname{Ker} \Delta \subset \mathbf{Q}\left[P_{F}^{1} \backslash 0,1, \infty\right]$ such that $\zeta_{F}(3)$ is equal to $\pi^{3 r_{2}} \cdot\left|d_{F}\right|^{-1 / 2}$ times the $\left(r_{1}+r_{2}\right)$-determinant $\| \mathscr{L}_{3}\left(\sigma_{j} y_{i} \| \cdot\left(1 \leq j \leq r_{1}+r_{2}\right)\right.$.

For $s=2$ a similar result was proved in [Z2]. It also follows directly from results of $[\mathrm{Bo}, \mathrm{B} 1, \mathrm{Su}]$. D. Zagier conjectured that an analogous fact should be valid for all integers $s \geq 3$ [Z3].

To prove Theorem 1 we give an explicit formula expressing the Borel regulator $r_{3}: K_{5}(\mathbf{C}) \rightarrow R$ by $\mathscr{L}_{3}(z)$, and then use the Borel theorem [Bo]. Below we indicate some ingredients of the proof which are of independent interest.

\section{Generic 3-VARIABLE fUNCTIONAL EQUATION FOR $\mathscr{L}_{3}(z)$}

The dilogarithm satisfies a remarkable 2-variable functional equation, discovered in the 19th century by W. Spence, N. H. Abel and others [L]. Its version for $D_{2}(z)$ is as follows. Let $r\left(x_{1}, \ldots, x_{4}\right)$ be the crossratio of a 4-tuple of different points on $P^{1}$. For every five different points on $P^{1}$ set

$$
\begin{aligned}
& R_{2}\left(x_{0}, \ldots, x_{4}\right):= \\
& \quad \sum_{i=0}^{4}(-1)^{i}\left[r\left(x_{0}, \ldots, \widehat{x}_{i}, \ldots, x_{4}\right)\right] \in \mathbf{Z}\left[P^{1} \backslash 0,1, \infty\right] .
\end{aligned}
$$

Then $D_{2}\left(R_{2}\left(x_{0}, \ldots, x_{4}\right)\right)=0$ in the sense of formula (1.4). Note that (3.1) depends actually on two variables because of the $P G L_{2}$ - 
invariance of the crossratio. It seems that any other functional equation for $D_{2}(z)$ can be deduced formally from this one.

It turns out that the analogous functional equation for $\mathscr{L}_{3}(z)$ corresponds to a special configuration of seven points in the plane. Namely, let $x_{1}, x_{2}, x_{3}$ be vertices of a triangle in $P_{F}^{2}$ (i.e. these points are not on a line); $y_{1}, y_{2}, y_{3}$ points on its "sides" $\overline{x_{1} x_{2}}$, $\overline{x_{2} x_{3}}$, and $\overline{x_{3} x_{1}}$, and $z$ a point in generic position (see Figure 1). Further, denote by $\left(y_{1} \mid y_{2}, y_{3}, x_{3}, z\right)$ the configuration of four points on a line obtained by projection of points $y_{2}, y_{3}, x_{3}, z$ with center at the point $y_{1}$. Set

$$
\begin{aligned}
R_{3}\left(x_{i}, y_{i}, z\right):= & \left(1+\tau+\tau^{2}\right) \\
& \circ\left[\left\{r\left(y_{1} \mid y_{2}, y_{3}, x_{2}, z\right)\right\}-\left\{r\left(y_{1} \mid y_{2}, y_{3}, x_{3}, z\right)\right\}\right. \\
& +\left\{r\left(z \mid x_{3}, y_{3}, x_{1}, y_{2}\right)\right\}+\left\{r\left(z \mid y_{3}, y_{1}, x_{1}, y_{2}\right)\right\} \\
& +\left\{r\left(z \mid y_{1}, x_{2}, x_{1}, y_{2}\right)\right\} \\
& \left.+\left\{r\left(z \mid x_{2}, x_{3}, x_{1}, y_{2}\right)\right\}-\left\{r\left(z \mid x_{3}, y_{1}, x_{1}, y_{2}\right)\right\}\right] \\
& +\left\{r\left(y_{1} \mid y_{2}, y_{3}, x_{2}, x_{3}\right)\right\}-3\{1\}
\end{aligned}
$$

where $\tau: x_{i} \rightarrow x_{i+1}, y_{i} \rightarrow y_{i+1}$ (indices modulo 3) (for example, $\left.\tau^{2} \circ\left\{r\left(y_{1} \mid y_{2}, y_{3}, x_{2}, z\right)\right\}=\left\{r\left(y_{3} \mid y_{1}, y_{2}, x_{1}, z\right)\right\}\right)$ and, by definition, $\{1\}=\{x\}+\{1-x\}+\left\{1-x^{-1}\right\}$ for any $x \in F^{*} \backslash 1$. As we will see below the choice of $x$ is inessential for our purposes.

Theorem 2. In the case $F=\mathrm{C}, \mathscr{L}_{3}\left(R_{3}\left(x_{i}, y_{i}, z\right)\right)=0$. Note, that $\mathscr{L}_{3}\left(\{x\}-\left\{x^{-1}\right\}\right)=0$ and $\mathscr{L}_{3}\left(\{x\}+\{1-x\}+\left\{1-x^{-1}\right\}\right)=\zeta_{\mathbf{Q}}(3)$.

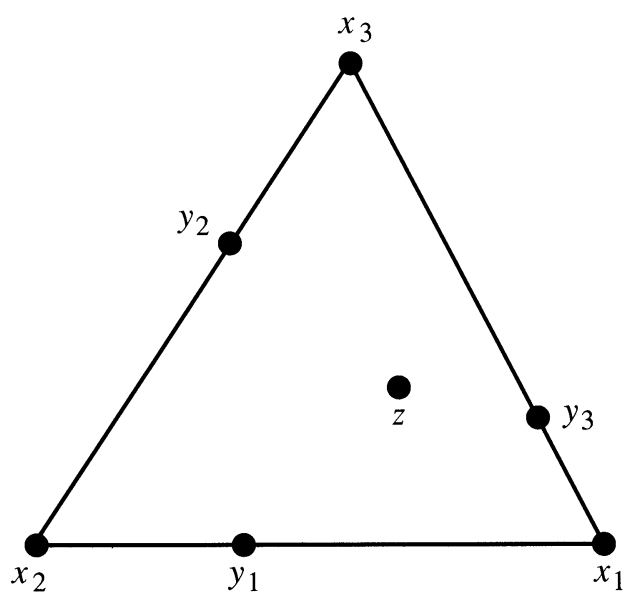

FIGURE 1 
A configuration $\left(x_{1}, x_{2}, x_{3}, y_{1}, y_{2}, y_{3}, z\right)$ of seven points in $P_{F}^{2}$ depends on three parameters. Consider a specialization of this configuration, when $z$ lies on the line $\overline{x_{3} y_{1}}$. It depends on two parameters, and the corresponding functional equation coincides with the classical Spence-Kemmer functional equation for the trilogarithm, discovered by Spence in 1809 [S] and, independently, by E. Kummer in 1840 [K] (see Chapter VI in [L]).

It is also possible to deduce the Spence-Kummer equation formally from Theorem 2 (as a linear combination of relations $\left.\mathscr{L}_{3}\left(R_{3}\left(x_{i}, y_{i}, z\right)\right)=0\right)$. The validity of the inverse statement is an interesting problem.

Conjecture 1. Any functional equation for $\mathscr{L}_{3}(z)$ can be formally deduced from Theorem 2.

\section{Algebraic $K$-THeORY OF A Field}

Now let $F$ be an arbitrary field. Set $B_{2}(F):=\mathbf{Z}\left[P_{F}^{1} \backslash 0,1, \infty\right]$ $/ R_{2}$, where $R_{2}$ is generated by elements $R_{2}\left(x_{0}, \ldots, x_{4}\right)$-see (3.1). Then there is the well-known Bloch complex $B_{2}(F) \stackrel{\delta}{\rightarrow}$ $\Lambda^{2} F^{*}$, where $\delta[x]=(1-x) \wedge x$. (It is not hard to prove that $\delta\left(R_{2}\right)=0$.). Thanks to Matsumoto, we know that Coker $\delta=$ $K_{2}(F)([M])$. Using some ideas of S. Bloch [B1], A. Suslin proved that $K_{3}^{\text {ind }}(F):=\operatorname{Coker}\left(K_{3}^{M}(F) \rightarrow K_{3}(F)\right)$ coincides with ker $\delta$ modulo torsion [Su].

Note also that $K_{1}(F)=F^{*}$ has an interpretation in the same spirit: $F^{*}=\mathbf{Z}\left[P_{F}^{1} \backslash 0,1, \infty\right] / R_{1}$, where $R_{1}$ is generated by expressions $[x]+[y]-[x y]$, reminiscent of the functional equation for $\ln |\cdot|$.

Let us define a complex $\mathbf{Q}(3)_{\mathscr{M}}$ as follows:

$$
\mathbf{Q}\left[P_{F}^{1} \backslash 0,1, \infty\right] / R_{3} \stackrel{\delta_{1}}{\rightarrow}\left(B_{2}(F) \otimes F^{*}\right)_{\mathbf{Q}} \stackrel{\delta_{2}}{\rightarrow}\left(\Lambda^{3} F^{*}\right)_{\mathbf{Q}}
$$

(the left group placed in degree 1), where $\delta_{2}[x] \otimes y=(1-x) \wedge x \wedge y$, $\delta_{1}\{x\}=[x] \otimes x$, and the subgroup $R_{3}$ is generated by $\{x\}-\left\{x^{-1}\right\}$, $\left(\{x\}+\{1-x\}+\left\{1-x^{-1}\right\}\right)-\left(\{y\}+\{1-y\}+\left\{1-y^{-1}\right\}\right)$ and $R_{3}\left(x_{i}, y_{i}, z\right)$ (see Equation 3.2).

Theorem $2^{\prime} . \delta_{1}\left(R_{3}\right)=0$ in $B_{2}(F) \otimes F^{*}$.

Hence the complex $\mathbf{Q}(3)_{\mathscr{M}}$ is well defined. Recall, that $K_{n}(F):=$ $\pi_{n}\left(B G L(F)^{+}\right)$, where $B G L(F)^{+}$is an $H$-space. Hence, by the Milnor-Moore theorem [MM] $K_{n}(F) \otimes \mathbf{Q}=\operatorname{Prim} H_{n}(G L(F), \mathbf{Q})$. 
A. Suslin proved [Su2] that $H_{n}\left(G L_{n}(F), \mathbf{Z}\right)=H_{n}(G L(F), \mathbf{Z})$. Therefore $K_{n}(F) \otimes \mathbf{Q}=\operatorname{Prim} H_{n}\left(G L_{n}(F), \mathbf{Q}\right)$. So $\operatorname{Im}\left(H_{n}\left(G L_{n-i}\right)\right.$ $\left.\rightarrow H_{n}\left(G L_{n}\right)\right)$ gives a canonical filtration $K_{n}(F)_{\mathbf{Q}} \supset K_{n}^{(1)}(F)_{\mathbf{Q}} \supset$ $\ldots$. Set $K_{n}^{[m]}(F)_{\mathbf{Q}}:=K_{n}^{(m)}(F)_{\mathbf{Q}} / K_{n}^{(m+1)}(F)_{\mathbf{Q}}$.

Theorem 3. There are canonical maps

$$
\begin{aligned}
& c_{1}: K_{5}^{[2]}(F)_{\mathbf{Q}} \rightarrow H^{1}\left(\mathbf{Q}(3)_{\mathscr{M}}\right) \\
& c_{1}: K_{4}^{[1]}(F)_{\mathbf{Q}} \rightarrow H^{2}\left(\mathbf{Q}(3)_{\mathscr{M}}\right) .
\end{aligned}
$$

Conjecture 2. $c_{1}$ and $c_{2}$ are isomorphisms.

Note, that according to [Su2]

$$
K_{3}^{[0]}(F)_{\mathbf{Q}} \simeq H^{3}\left(\mathbf{Q}(3)_{\mathscr{M}}\right) \equiv K_{3}^{M}(F)_{\mathbf{Q}}
$$

(A. A. Beilinson and S. Lichtenbaum conjectured that there should exist complexes $\mathbf{Q}(j)_{\mathscr{M}}$ computing all $K_{n}(F)$-see [Be2, Li].)

\section{THE GROUP $B_{3}(F)$}

For a $\mathbf{G}$-space $X$, points of $G \backslash X \times \ldots \times X$ are called configurations. Let $\mathbf{Z}\left(C_{6}\left(P_{F}^{2}\right)\right)$ be the free abelian group generated by all possible configurations $\left(l_{0}, \ldots, l_{5}\right)$ of 6 points in $P_{F}^{2}$.

Let us define a homomorphism $L_{3}: \mathbf{Z}\left[P_{F}^{1} \backslash 0,1, \infty\right] \rightarrow$ $\mathbf{Z}\left[C_{6}\left(P_{F}^{2}\right)\right]$ as follows: $L_{3}\{x\}=\left(x_{1}, x_{2}, x_{3}, y_{1}, y_{2}, y_{3}\right)$, where $r\left(y_{1} \mid x_{1}, x_{2}, y_{2}, y_{3}\right)=x$ (this configuration was described in $\S 3$ ). The (unique) configuration where $y_{1}, y_{2}, y_{3}$ are on a line will be denoted $\eta_{3}$.

Definition. $B_{3}(F)$ is the quotient of the group $\mathrm{Z}\left[C_{6}\left(P_{F}^{2}\right)\right]$ by the following relations

(R1) $\left(l_{0}, \ldots, l_{5}\right)=0$, if two of the points $l_{i}$ coincide or four lie on a line.

(R2) (The seven-term relation.) For any seven points $\left(l_{0}, \ldots, l_{6}\right)$ in $P_{F}^{2}$

$$
\sum_{i=0}^{6}(-1)^{i}\left(l_{0}, \ldots, \hat{l}_{i}, \ldots, l_{6}\right)=0 .
$$


(R3) Let $\left(m_{0}, \ldots, m_{5}\right)$ be a configuration of six points in $P_{F}^{2}$, such that $m_{2}=\overline{m_{0} m_{1}} \cap \overline{m_{3} m_{4}}$ and $m_{5}$ is in generic position-see Fig. 2 . Then if $L_{3}^{\prime}\{x\}:=-L_{3}\{x\}-2 L_{3}\{1-x\}$, $\left(m_{0}, \ldots, m_{5}\right)$

$$
=\frac{1}{3} \sum_{i=0}^{4}(-1)^{i} L_{3}^{\prime}\left\{r\left(m_{5} \mid m_{0}, \ldots, \widehat{m}_{i}, \ldots, m_{4}\right)\right\}+\frac{1}{3} \eta_{3} .
$$

Lemma. In the group $B_{3}(F)$ we have

$$
\left(l_{0}, \ldots, l_{5}\right)=(-1)^{|\sigma|}\left(l_{\sigma(0)}, \ldots, l_{\sigma(5)}\right) .
$$

Remark. The configurations from (R1) are just the unstable ones in the sense of D. Mumford.

Theorem 4. The homomorphism $L_{3}: \mathbf{Z}\left[P_{F}^{1} \backslash 0,1, \infty\right] \rightarrow \mathbf{Z}\left[C_{6}\left(P_{F}^{2}\right)\right]$ induces an isomorphism modulo 6-torsion.

$$
L_{3}: \mathbf{Z}\left[P_{F}^{1} \backslash 0,1, \infty\right] / R_{3} \underset{\rightarrow}{\sim} B_{3}(F) \otimes \mathbf{Z} .
$$

(It is easy to check using (R2) and (R3) that $L_{3}$ is onto; the 7term relation for a configuration $\left(x_{1}, x_{2}, x_{3}, y_{1}, y_{2}, y_{3}, z\right)$ then coincides with $\left(L_{3}\left(R_{3}\left(x_{i}, y_{i}, z\right)\right)\right.$.)

Let us denote by $M_{3}$ the inverse homomorphism. Then the composition $L_{3} \circ M_{3}: B_{3}(\mathbf{C}) \rightarrow \mathbf{Q}\left[P_{\mathbf{C}}^{1} \backslash 0,1, \infty\right] \rightarrow \mathbf{R}$ defines a measurable function on configurations of six points in $\mathbf{C} P^{2}$, satisfying functional relations (R1) through (R3). So for $x \in P_{\mathbf{C}}^{2},\left(L_{3}\right.$ 。 $\left.M_{3}\right)\left(x, g_{1} x, \ldots, g_{5} x\right)$ is a measurable cocycle. Let us prove that its cohomology class lies in $\operatorname{Im}\left(H_{\mathrm{cts}}^{5}\left(G L_{3}(\mathbf{C}), R\right) \rightarrow H^{5}\left(G L_{3}(\mathbf{C}), R\right)\right)$, where $H_{\mathrm{cts}}^{*}(G, R)$ is continuous cohomology.

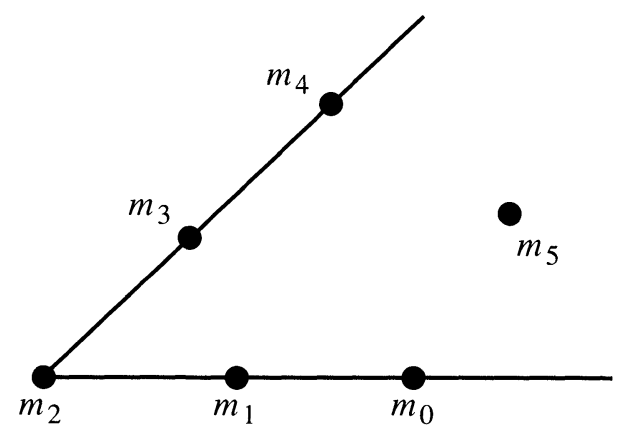

FIGURE 2 
Consider the complex

Meas $C_{2 n-1}\left(\mathbf{C} P^{n-1}\right) \stackrel{d_{2 n-1}^{*}}{\rightarrow}$ Meas $C_{2 n}\left(\mathbf{C} P^{n-1}\right) \stackrel{d_{2 n}^{*}}{\rightarrow}$ Meas $C_{2 n+1}\left(\mathbf{C} P^{n-1}\right)$

where $C_{m}\left(\mathbf{C} P^{n}\right)$ is the space of all configurations of $m$ points in $\mathbf{C} P^{n}, \operatorname{Meas}(X)$ is the space of all measurable functions on the space $X, d_{m}:\left(l_{0}, \ldots, l_{m}\right) \mapsto \sum_{i=0}^{m}(-1)^{i}\left(l_{0}, \ldots, \hat{l}_{i}, \ldots, l_{m}\right)$ and $d_{m}^{*}$ is the induced map.

Theorem 5. $\operatorname{Ker} d_{2 n}^{*} / \operatorname{Im} d_{2 n-1}^{*}$ is canonically isomorphic to the indecomposable part of $H_{\mathrm{cts}}^{2 n-1}\left(G L_{n}(\mathbf{C}), R\right)$.

For $n=2$ this was proved in [B1]. See also closely related work [HM].

Conjecture 3. There exists a canonical element in $\operatorname{Ker} d_{2 n}^{*}$ that can be expressed by classical $n$-logarithm $\mathscr{L}_{n}(z)$ and represents the Borel class in $H_{\mathrm{cts}}^{2 n-1}\left(G L_{n}(\mathbf{C}), R\right)$.

I would like to thank A. A. Beilinson for stimulating discussions and interest and M. L. Kontsevich for useful remarks.

\section{REFERENCES}

[Be1] A. A. Beilinson, Polylogarithm and cyclotomic elements, preprint 1989.

[Be2] _ Height pairing between algebraic cycles, Lecture Notes in Math., vol. 1289, Springer, New York, pp. 1-26.

[B1] S. Bloch, Higher regulators, algebraic $K$-theory and zeta functions of elliptic curves, Lecture Notes, University of California, Irvine, 1977.

[Bo] A. Borel, Cohomology de $S L_{n}$ et valeurs de functions zeta aux points entiers, Ann. Scuola Norm. Sup. Pisa Cl. Sci. (4) 4, (1977), 613-636.

[HM] R. Hain and R. MacPherson, Higher logarithms, preprint 1989.

[K] E. E. Kummer, J. Pure Appl. Math., (Crelle) 21 (1840).

[L] L. Lewin, Polylogarithms and associated functions, North-Holland, New York, 1981.

[Li] S. Lichtenbaum, Values of zeta functions at non-negative integers, Lecture Notes in Math., vol. 1086, Springer-Verlag, Berlin and New York, 1984, pp. 127-138.

[M] J. Milnor, Introduction to algebraic K-theory, Princeton, N.Y., 1971.

[MM] J. Milnor and J. Moore, On the structure of Hopf algebras, Ann. of Math. (2) 81 (1965), 211-264.

[R] D. Ramakrishnan, Analogs of the Bloch-Wigner function for higher polylogarithms, Contemp. Math., Amer. Math. Soc., Providence, R.I., vol. 55, 1986, pp. 371-376.

[S] W. Spence, An essay on logarithmic transcendents, London and Edinburgh, 1809, pp. 26-34. 
[Su] A. A. Suslin, Algebraic $K$-theory of fields, Proc. of the International Congress of Mathematicians, 1986, Amer. Math. Soc., Providence, R.I., 1987, pp. 222-244.

[Su2] _ Homology of $G L_{n}$, characteristic classes, and Milnor $K$-theory, Lecture Notes in Math., vol. 1046, Springer-Verlag, New York, 1984, pp. 357375.

[Z] D. Zagier, The Bloch-Wigner-Ramakrishnan polylogarithm function, Math. Ann. 286 (1990), 613-640.

[Z2] __ Hyperbolic manifolds and special values of Dedekind zeta-functions, Invent. Math. 83 (2) (1986), 285-301.

[Z3] _ Polylogarithms, Dedekind zeta functions, and the algebraic $K$-theory of fields, Proceedings of the Texel Conference on Arithmetic Algebraic Geometry, Birkhäuser, Boston (to appear).

SCIENTific Council for Cybernetics, Moscow, USSR 